\title{
Adipolin and insulin resistance response to two types of exercise training in type 2 diabetic male rats
}

\begin{abstract}
Introduction: Adipolin (CTRP12), which improves insulin sensitivity, is a novel antiinflammatory adipocytokine secreted from adipose tissue. In this study we aimed to assess the Adipolin and Insulin Resistance index (HOMA-IR) response to two types of exercise in type 2 diabetic male rats.

Materials and methods: In this study, 24diabetic Wistar rats (Induced by high-fat diet and Intraperitoneal injection Streptozotocin (stz) injection) were randomly assigned to 3 groups: High intensity interval training (HIIT), low intensity continuous training (LICT) and control (C). Both training groups were trained on the treadmill, 5 sessions per week for 8 weeks. Blood samples were taken 24 hours after the end of training session and plasma adipolin, insulin and glucose levels were measured. ANOVA and Tukey post hoc tests were used to analyze data and the level of significance was considered to $b e \mathrm{p} \leq 05$.

Results: Data analysis showed that plasma adipolin levels in the low intensity continuous training group were significantly increased, compared to the control group $(\mathrm{p}=0.006)$. Plasma glucose level in both the low intensity continuous training and the high intensity interval groups was significantly decreased, compared to the control group $(p=0.049)$ \& $(\mathrm{p}=0.007)$. Plasma insulin level in both training groups was increased and HOMA-IR index was decreased, compared to the control group, although changes were not significant.
\end{abstract}

Conclusion: The results of this study showed that exercise training can increase plasma adipolin in rats with type 2 diabetes, changes however that are partially dependent on the type of exercise training

Keywords: high intensity interval training, low intensity continous training, adipolin, insulin resistance, type 2 diabetes
Volume 6 Issue I - 2018

\author{
Mina Rahmatollahi,' Ravasi AA,' Soori R,' \\ Onegh B, ${ }^{2}$ Dolati $F^{3}$ \\ 'Faculty of Physical Education and Sport Sciences, University of \\ Tehran, Iran \\ 2Department of Biological Chemistry, Faculty of Chemistry, \\ University of Tarbiat Modarres, Iran \\ ${ }^{3}$ Masrers of Physical Education and Sport Sciences, Azad \\ University of Tehran, Iran
}

Correspondence: Mina Rahmatollahi, Faculty of Physical Education and Sport Sciences, University of Tehran, Center, Tehran, IR, Iran, Email targol_0169@yahoo.com

Received: October 18,2017 | Published: January 3I, 2018
Abbreviations: HIIT, high intensity interval training; LICT, low intensity continuous training; ER, endotracheal stress; HITT, high intensity interval training

\section{Introduction}

Regular exercise is a good strategy for treating many metabolic disorders, including type 2 diabetes and obesity. Some part of the beneficial effects of exercises are due to endocrine exercise, including fatty tissue which plays an important role in regulating energy metabolism, body composition, and insulin resistance. ${ }^{1}$ The adipose tissue, as an active endocrine and paracrine tissue as well as because of its role in the synthesis and secretion of a series of hormones and adipocytokines, , not only controls body weight balance, but also Justifies the relationship between overweight and obesity with insulin resistance and diabetes by effecting the metabolic and inflammatory profile., ${ }^{2,3}$ Recently, a new adipocytokine been identified called Adipolin (CTRP12) which is an anti-inflammatory cytokine that is synthesized and secreted mainly in adipose tissue and decreases in obesity and diabetes. ${ }^{3,4}$. Additionally, adipolin also helps to improve insulin sensitivity. ${ }^{3,4}$ Adipolin is found in blood circulation in intact (fCTRP12) $(40 \mathrm{kDa})$ and broken (spherical) (gCTRP12) $(25 \mathrm{kDa})$ forms. ${ }^{4}$ Studies have shown that only fCTRP12 isophoresis of adipolin can improve insulin resistance. ${ }^{5}$ Therefore, any factor that affects the expression of the gene and the synthesis of adipolin, or in the breakdown of adipolin and the reduction of its intact form, can reduce insulin sensitivity, as well as insulin that mainly breaks the fCTRP12.5 Therefore, lowering insulin levels may be one of the effective ways to improve adipollin function. Many therapeutic strategies are known to reduce insulin in obesity and insulin resistance. Physical activity is one of these most important factors. Thus, it is likely that physical activity and exercise help to improve Insulin resistance not only by making direct effect on insulin and its performance, but also through their effect on adipolin levels and changing the ratio of intact adipolin to broken forms. But according to our knowledge, the effect of exercise training on adipolin levels has not been studied in any of the present studies. Adipolin levels are reduced in diabetic patients, while exercise may reduce adipollin-induced diabetic circulation, and this effect is likely to depend on a type of exercise that is performed in varying degrees. However, this hypothesis has not been studied in diabetic specimens with lower adipolin base levels. Therefore, the present study was conducted to evaluate the response of adipolin plasma to high intensity interval training (HITT) and low intensity continuous training (LICT) in male rats with type II diabetes and the present study seeks to answer the question whether low and high intensity continous training have a significant effect on plasma Adipolin, insulin, fasting glucose, and resistance index in diabetic male rats?

\section{Methods and materials}

The present study is an experimental design with a post-test design with the present of a control group. 24 Wistar male rats with the age range of 6 weeks and weighing $110 \pm 10 \mathrm{~g}$ were purchased from Pasteur 
Institute of Iran and were transferred to the animal house of the Faculty of Physical Education and Sports Sciences of Tehran University in accordance with the policy of the Iranian Association for the Protection of lab animals used for scientific and laboratory purposes. All rats were kept in cages of four rat capacity under controlled environmental conditions with an average temperature of $22 \pm 2^{\circ} \mathrm{C}$, a day-night cycle of $12: 12$ hours, a relative humidity of $50 \%$ and with free access to water and special diet of rats. For this purpose, After familiarity and adaptation to the new environment, the rats exposed to a high-fat diet for 8 weeks (prepared by the Razi Serum Institute), which contained $45 \%$ of the total energy of fat (derived from animal fat) that contains 24grams of fat, 24grams of protein and 41 grams of carbohydrates per 100 grams. ${ }^{6}$. After 8 weeks, diabetes induction was conducted with single dose of Streptozotocin (STZ) dissolved in sodium citrate buffer with a $\mathrm{pH}$ of $4.5 \mathrm{mg} / \mathrm{kg}(30 \mathrm{mg} / \mathrm{kg})$ by intraperitoneal (IP) method. ${ }^{6}$ To confirm diabetes, 96hours after injection, a small drop of blood in the tail of animals was placed on a glucometric strip and was read by the glucometer and blood glucose levels above $300 \mathrm{~m} . \mathrm{g} / \mathrm{dL}$ were considered as an indicator of diabetic situation. ${ }^{6}$ The rats were randomly divided into three groups of high intensity interval training (HITT) (7rats), low intensity continuous training (LICT) (7rats) and control (6rats) after being sure of being diabetic. Also, four other rats were selected as pilot groups for measuring maximum running speed on treadmill. In order to estimate maximum running speed, we performed a graded sport performance with a zero-degree gradient that started at $10 \mathrm{~m} / \mathrm{min}$ and the speed of treadmill was increased by $1 \mathrm{~m} / \mathrm{min}$ for 1 minute so that the rats could not run (exhaustion). After estimating the maximum speed, each of the two training groups (HITT) and (LICT activated on the treadmill 5 sessions per week and for 8weeks. The protocol (HITT) was the implementation of $80-85 \%$ maximum speed training for 2 minutes with 1 minute rest periods, which reached 12 in the final week from 6 times in the first week. ${ }^{7,8}$ 48 hours after the last exercise session, all rats were anesthetized with a combination of ketamine $(75 \mathrm{mg} / \mathrm{kg})$ and xylacin $(10 \mathrm{mg} / \mathrm{kg})$. After ensuring animal anesthesia, the chest of the animal was split and about $10 \mathrm{ml}$ of blood was taken directly from the animal's heart and pured in to tubes containing EDTA. The collected samples were centrifuged for 15 minutes at a speed of 3,000rpm and their plasma was separated, then transferred to a $-80^{\circ} \mathrm{C}$ freezer temperature for further evaluations. Plasma Adipolin was measured using ELISA Kit (family with sequence similarity 132, member A) from My Bio Sourse Inc, USA. The sensitivity of the measurement was $0.1 \mathrm{ng} / \mathrm{ml}$. The plasma glucose concentration was measured by glucose oxidase enzyme-colorimetric method using glucose kit (Pars Test, Iran). The coefficient of variation and sensitivity of the measurement method were $1.8 \%$ and $5 \mathrm{mg} / \mathrm{ml}$ respectively. Plasma insulin measurement was performed by ELISA method using Mercodia Rat Insulin ELISA kit (constructed in Sweden) with sensitivity of $0.07 \mathrm{ig} / \mathrm{dl}$. The index of insulin resistance was also calculated by the HOMA-IR method using the following formula. $\mathrm{P} \leq 0.05$ was considered significant.

HOMA formula (insulin resistance index)=Fasting glucose (mg/ $\mathrm{dL}$ ) $\times$ Fasting insulin (micro unit per $\mathrm{ml}$ ) $/ 405$. The data were analyzed by SPSS software version 22 at the significance level $(\mathrm{P} \leq 0.05)$. After the data were normalized by Kolmogorov-Smirnov test (KS), one way Anova and Tukey methods were used to determine the significance of variables between the groups.

\section{Findings}

Body weight changes between the research groups at different stages of the study are shown in Table 1 and the mean and standard deviation of the variables of the research are presented in Table 2.

Table I The mean and standard deviation of the weight of rats in the research groups during the study (g)

\begin{tabular}{lllll}
\hline The level//group & Initial & $\begin{array}{l}\text { After 8weeks of high } \\
\text { fat diet }\end{array}$ & $\begin{array}{l}\text { After diabetes } \\
\text { induction }\end{array}$ & Final \\
\hline Control & $176.66 \pm 7.17$ & $345.16 \pm 3.71$ & $251.33 \pm 42.69$ & $223.83 \pm 55.70$ \\
High intensity interval Training & $176.83 \pm 8.13$ & $332 \pm 11.36$ & $246.83 \pm 5.84$ & $235.16 \pm 7.83$ \\
Low intensity continous Training & $177.33 \pm 7.42$ & $340.66 \pm 7.33$ & $259.66 \pm 20.45$ & $242.83 \pm 3.07$
\end{tabular}

Table 2 The mean and standard deviation of the variables in research groups

\begin{tabular}{lll}
\hline Variable/group & Insulin (micro unit/bpm) & Insulin resistance index \\
\hline Control & $4.62 \pm 0.58$ & $4.15 \pm 0.58$ \\
High intensity interval training & $4.74 \pm 0.39$ & $3.58 \pm 0.36$ \\
Low intensity continous training & $4.87 \pm 0.32$ & $3.84 \pm 0.30$ \\
\hline
\end{tabular}

*Significance compared to the control group $(\mathrm{p} \leq 0.05)$

Plasma adipoline levels were significantly different between the groups $(\mathrm{P}=0.000, \mathrm{~F}=8027)$. Tukey test results showed that plasma adipoline levels were significantly increased in the LICT group compared with the control group $(\mathrm{P}=006)$. Adipolin levels increased in the high intensity training group, but this increase was not significant and there was no difference between the other groups. Plasma adipoline levels of different groups are presented in Figure 1. Also, the findings of the present study showed that there was no significant difference in plasma insulin and insulin resistance index among the study groups $(\mathrm{p}<0.05)$, however, the difference in plasma glucose was significant $(p<0.0001, F=113.56)$, so both types of high and low intensity training caused a significant reduction in plasma glucose levels $(\mathrm{p}=0.049)$ and $(\mathrm{p}=0.007)$ and there was no difference between the two training groups. Plasma glucose levels between different groups are shown in Figure 2. Also, the correlation between the data is given in Table 3.

Chart $1 \& 2$ 
Table 3 Correlation between the data

\begin{tabular}{|c|c|c|c|c|c|c|c|}
\hline Variables & Adipolin & $\begin{array}{l}\text { Insulin } \\
\text { resistance index }\end{array}$ & Glucose & Insulin & $\begin{array}{l}\text { Istweek } \\
\text { weight }\end{array}$ & $\begin{array}{l}9^{\text {th }} \text { week } \\
\text { weight }\end{array}$ & $16^{\text {th }}$ week weight \\
\hline Adipolin & I & 0.289 & 0.315 & -0.332 & $0.876^{*}$ & -0.388 & 0.089 \\
\hline Insulin Resistance index & 0.289 & I & $0.959 \S$ & $-0.560^{\S}$ & 0.544 & $-0.776^{\S}$ & 0.219 \\
\hline flucose & 0.315 & $0.959 \S$ & I & $-0.747^{\S}$ & 0.543 & $-0.8 \mid 4^{\S}$ & -0.002 \\
\hline Insulin & -0.332 & $-0.560 \S$ & $-0.747^{\S}$ & I & 0.483 & $0.702 \S$ & 0.276 \\
\hline$\left.\right|^{\text {st }}$ week weight & $0.876 *$ & 0.544 & 0.543 & 0.483 & I & $0.912 *$ & $\mathrm{a}$ \\
\hline $9^{\text {th }}$ week weight & -0.388 & $-0.766^{\S}$ & $-0.8 \mid 4^{\S}$ & $0.702^{\S}$ & $0.92 *$ & I & $0.875^{\S}$ \\
\hline $16^{\text {th }}$ week weight & 0.089 & 0.219 & -0.002 & 0.276 & $\mathrm{a}$ & 0.875 & I \\
\hline
\end{tabular}

*Correlation at the level of 0.05 was significant. $\S$ : Correlation at the level of 0.01 was significant. a: is the lack of correlation due to the fixed constant of one variable

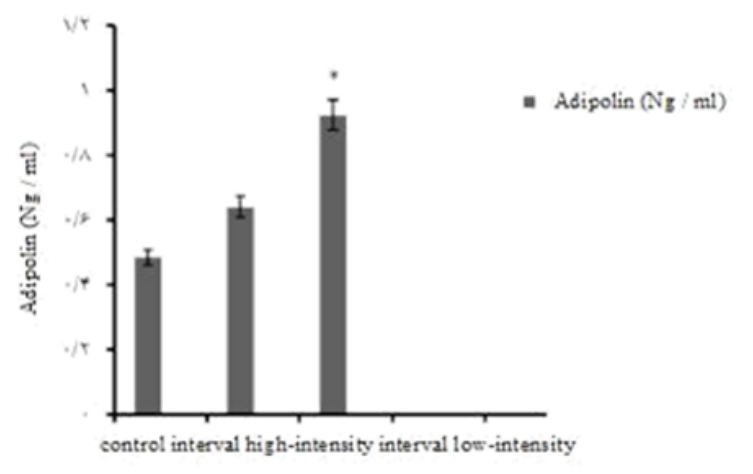

Chart I Plasma adicolin levels in different groups. *Significance compared to the control group.

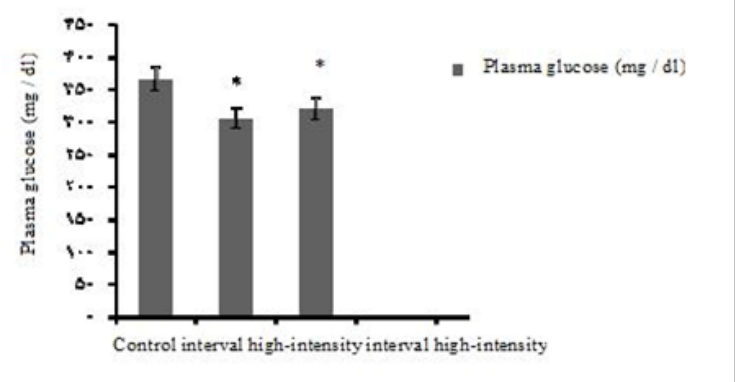

Chart 2 Plasma glucose levels in different groups.

*Significance compared to the control group.

\section{Discussion}

According to the results of this study, the implementation of 8 weeks of low-intensity continous training (LICT) increased serum adipolin which is significant statistically $(\mathrm{p}=0.006)$ and despite the increase in plasma adipolin levels due to High intensity interval training (HIIT), these changes were not statistically significant. Also, there was no significant difference between the two types of training protocols HIIT with LICT in adipolin plasma. Since no studies have evaluated the effect of any type of exercise or trainings on the levels of adipolin in diabetic individuals, the investigator justifies adipolin changes after 8 weeks of intense interval and continuous trainings based on theoretical foundations. Gene expression and serum adipolin levels decrease in obese human and animal samples. ${ }^{3}$ In fact, adipolin expression is under the negative control of obesity-related stress. So, by inducing TNF- $\alpha$ and endoplasmic stress in the adipose cell culture medium, adipolin expression declines. ${ }^{3}$ TNF- $\alpha$ is an adipocyte-derived anti-inflammatory adipocytokine, ${ }^{3}$ which decreases following low-intensity continuous exercise and weight loss. ${ }^{9}$ TNF- $\alpha$ is capable in affecting some of the translation factors and thud influencing metabolism of adipoline levels; KLF-15 is one of these factors. KLF-15 is a member of the large family of KLF transcription factors that contribute to the regulation of glucose metabolism and adipogenesis. ${ }^{10,11}$ Enomoto et al. have observed that, as adipolin, the expression of KLF-15 is also lower in the lipid profile of DIO mice compared to the control group. ${ }^{12}$ Since TNF- $\alpha$ increases the expression of pre-inflammatory cytokines by activating JNK in adipocytes, ${ }^{12-16}$ so increases the insulin resistance induced by obesity and thereby provides the suitable conditions for the occurrence or exacerbation of insulin resistance. Endoplasmic Endotracheal Stress (ER) is also an inflammatory factor that interacts with obesity and type II diabetes and helps the spread of inflammation, apoptosis of pancreatic beta cells, insulin synthesis disorder and insulin resistance. ${ }^{17}$ and decreases Adipolin expression in cultured adipose cells. ${ }^{3}$ Since exercise is one of the proposed therapies for the improvement of inflammation and the prevention and treatment of obesity and metabolic disorders associated with type II diabetes and insulin resistance, some studies aimed at identifying intermediary molecular mechanisms effected by physical activity and training in this area, have examined the effects of different types of training protocols on TNF- $\alpha$ and ER-related molecules. Studies have shown that TNF- $\alpha$ levels decreases following continous low intensity training and weight loss. ${ }^{9}$ Additionally, compatibility with sport exercises, while improving the inflammation in obese subjects, inhibits ER stress and improves UPR. ${ }^{18}$ Although 
the present study did not measure the levels of TNF- $\alpha$ and biomarkers associated with ER or UPR, but due to the relationship between TNF- $\alpha$ and biomarkers associated with ER or UPR stress with changes in obesity, it is expected to reduce weight, body mass index and body fat percentage after 8 weeks of low intensity trainingd significantly. However, due to the lack of alignment of adipoline plasma levels with changes in body weight after 8 weeks of training, non-obesity factors are likely to affect adipolin levels after trainings, which are less probable to be affected by weight loss following exercise exercises; insulin can be one of these candidates. ${ }^{19}$ Insulin is the hormone regulating the carbohydrate metabolism and glucose hemostasis which increases the transfer of GLUT4 to the cell membrane and transfusion tubes and increases glucose clearance by binding. ${ }^{20} \mathrm{~g}$ to the alphareceptor insulin subunit and triggering the insulin signal pathway The regulatins effect of insulin on adipolin is two dimentionalm, in the lean subject,by activating the PI3K pathway can increase the expression and secretion of adipoline,${ }^{21}$ but in obese person that is also susceptible to insulin resistance, this hemostatic interaction between insulin and adipolin stops and insulin reduces the levels of adipolin. ${ }^{22,23}$ In this study, plasma glucose levels and HOMA-IR values decreased after 8 weeks of continuous low-intensity exercise, and this change was statistically significant $(p=0.009)$. Training modulates Insulin Resistance through: 1) insulin receptor; 2) increasing protein and mRNA carriers of glucose (GLUT4); 3) increasing glycogen synthase, protein kinase-B and hexokinase; 4) improving intracellular insulin messaging and effecting on intermediate molecules in an insulin signal such as increasing the expression of ERK2, increasing the activity of PI3K or Akt / PKB, and improving the AMPK signal; (27); ${ }^{5}$ changing in muscle composition (increasing capillary density in muscle fibers and conversion of muscle fibers to oxidative fasting fibers); 6) increasing the delivery of glucose to muscle; 7) reducing triglyceride accumulation in muscle cells; and 8) reducing the release of fatty acids and increasing their oxidation and purification..$^{24}$ The two characteristics of the intensity and duration of the trainings strongly influence the response of insulin exercise, so that the improvement of insulin sensitivity occurs when the amount of exercise is at its highest level. ${ }^{25}$ Since the subjects in the present study included obese male rats and because of this, there is no limitation in the design of training characteristics, the intensity, duration and volume of exercises appropriate with the above mediators of each pathway can be used to modify the levels of insulin, glucose and HOMA-IR. Regarding the inverse relationship between insulin and glucose with adipolin, a significant change in insulin and glucose can be one of the causes of a significant change in adipoline after 8 weeks of low intensity training.

Despite the limitations, such as the lack of measurement of TNF- $\alpha$ levels and endometrial stress-associated biomarkers (UPR), the results of this study suggest that exercising can increase plasma adipolysis in type 2 diabetic rats. However, these changes are partly related to training's type and it seems that increasing adipoline as a result of exercise can be an effective factor in improving plasma glucose in type 2 diabetes rats. Since this study is among the first studies conducted on the subject of the response of adipolin to both low and high intensity continuous trainings, further studies are needed to understand the mechanism associated with this.

\section{Acknowledgements}

The present study is based on a research design at the Faculty of Physical Education and Sports Sciences of the University of Tehran without any financial support. Thanks and appreciation to everyone who collaborated on the research.

\section{Conflicts of interest}

None.

\section{Funding}

None.

\section{References}

1. Welc SS, Clanton TL. The regulation of interleukiný6 implicates skeletal muscle as an integrative stress sensor and endocrine organ Exp Physiol. 2013;98:359-371.

2. Borges-Silva CN, Fonseca-Alaniz MH, Alonso-Vale MI, et al. Reduced lipolysis and increased lipogenesis in adipose tissue frompinealectomized rats adapted to training. $J$ Pineal Res. 2005;39(2):178-184.

3. Enomoto T, Ohashi K, Shibata R, et al. Adipolin/C1qde2/CTRP12 metabolism. J Biol Chem. 2011;286(40):34552-34558.

4. Wei Z, Peterson JM, Lei X, et al. C1q/TNF- related protein-12 (CTRP12,) a novel adipokine that improves insulin sensitivity and glycemic control in mouse models of obesity and diabetes. $J$ Biol Chem. 2012;287(13):10301-10315.

5. Wei Z, Lei X, Seldin MM, et al. Endopeptidase cleavage generates a functionally distinct isoform of $\mathrm{C} 1 \mathrm{q} /$ tumor necrosis factor- related protein-12 (CTRP12) with an altered oligomeric state and signaling specificity. J Biol Chem. 2012;287(43):35804-35814.

6. Holmes A, Coppey LJ, Davidson EP, et al. Rat models of diet-induced obesity and high fat/low dose streptozotocin type 2 diabetes: effect of reversal of high fat diet compared to treatment with enalapril or menhaden oil on glucose utilization and neuropathic endpoints. Journal of Diabetes Research. 2015;2015(2015):8.

7. Thomas C, Bishop D, Moore-Morris T, et al. Effects of high-intensity training on MCT1, MCT4, and $\mathrm{NBC}$ expressions in rat skeletal muscles: influence ofchronic metabolic alkalosis. Am J Physiol Endocrinol Metab. 2007;293(4):E916-E922.

8. Pereira F, de Moraes R, Tibiriçá E, et al. Continous and continuous exercise training produce similar increases in skeletal muscle and left ventricle microvascular density in rats. BioMed research international. 2013;2013(2013):7.

9. Soori R, Rezaeian N, Salehian O. Effects of high and low intensity endurance training on levels of leptin, cortisol, testosterone, growth hormone, and insulin resistance index in sedentary obese men. J Sport Biomotor Sci. 2012;6:17-28.

10. Sohaily SH, Soori R, Rezaeian N. Hormonal adaptations to moderateintensity endurance training in sedentary obese men. Koomesh. 2013;14(2):181-191.

11. Goldberg DP, Hillier VF. A scaled version of the Gen eral Health Questionnaire. Psychol Med. 1979;9(1):139-145.

12. Soori R, Rezaeian N, Salehian O. Effects of Continous Training on Leptin and Hormone levels Affecting Lipid Metabolism in Young Obese/ Overweight Men. Iran J Endocrinol Metabolism. 2012;14(3):248-256

13. Mirmiran P, Esfahani FH, Mehrabi Y, et al. Reliability and relative validity of an FFQ for nutrients in the Tehran lipid and glucose study. Public Health Nutr. 2010;13(5):654-662.

14. Tanaka H, Monahan KD, Seals DR. Age-predicted maximal heart rate revisited. J Am Coll Cardiol. 2001;37(1):153-156.

15. Jackson AS, Pollock ML. Generalized equations for predicting body density of men. Br J Nutr. 1978;40(3):497-504. 
16. Siri WE. Body composition from fluid spaces and density:analysis of methods. 1961. Nutrition. 1993;9(5):480-491.

17. Lau DC, Douketis JD, Morrison KM, et al. Obesity Canada Clinical Practice Guidelines Expert Panel. 2006 Canadian clinical practice guidelines on the management and prevention of obesity in adults and children [summary]. CMAJ. 2007;176(8):S1-S13.

18. Nieman DC, Brock DW, Butterworth D, et al. Reducing diet and/or exercise training decreases the lipid and lipoprotein risk factors of moderately obese women. J Am Coll Nutr. 2002;21(4):344-350.

19. Ciolac EG, Brech GC, Greve JM. Age does not affect exercise intensity progression among women. $J$ Strength Cond Res. 2010;24(11):3023-3031.

20. Matthews DR, Hosker JP, Rudenski AS, et al. Homeostasis model assessment: insulin resistance and beta-cell function from fasting plasma glucose and insulin concentrations in man. Diabetologia. 1995;28(7):412-419.
21. Balducci S, Zanuso S, Nicolucci A, et al. Anti-inflammatory effect of exercise training in subjects with type 2 diabetes and the metabolic syndrome isdependent on exercise modalities and independent of weight loss. Nutr Metab Cardiovasc Dis. 2010;20(8):608-617.

22. Pearson R, Fleetwood J, Eaton S, et al. Krüppel-like transcription factors: a functional family. Int J Biochem Cell Biol. 2008;40(10):1996-2001.

23. Mori T, Sakaue H, Iguchi H, et al. Role of Kruppel-like factor 15 (KLF15) in transcriptional regulation of adipogenesis. J Biol Chem. 2005;280(13):12867-12875.

24. Enomoto T, Ohashi K, Shibata R, et al. Transcriptional regulation of an insulin-sensitizing adipokine adipolin/CTRP12 in adipocytes by Krüppel- likefactor 15. PLoS One. 2013;8(12):e83183.

25. Kawamata Y, Imamura T, Babendure JL, et al. Tumor necrosis factor receptor-1 can function through a $G$ alpha $q / 11$-betaarrestin-1 ssignaling complex. J Biol Chem. 2007;282(39):28549-2856. 